\title{
Hierarchical structure observation and nanoindentation size effect characterization for a limnetic shell
}

\author{
Jingru Song ${ }^{1} \cdot$ Cuncai Fan $^{1} \cdot$ Hansong $\mathrm{Ma}^{1} \cdot$ Yueguang Wei $^{1}$
}

Received: 14 November 2014 / Revised: 18 December 2014 / Accepted: 12 January 2015 / Published online: 26 May 2015

(C) The Chinese Society of Theoretical and Applied Mechanics; Institute of Mechanics, Chinese Academy of Sciences and Springer-Verlag Berlin Heidelberg 2015

\begin{abstract}
In the present research, hierarchical structure observation and mechanical property characterization for a type of biomaterial are carried out. The investigated biomaterial is Hyriopsis cumingii, a typical limnetic shell, which consists of two different structural layers, a prismatic "pillar" structure and a nacreous "brick and mortar" structure. The prismatic layer looks like a "pillar forest" with variationsection pillars sized on the order of several tens of microns. The nacreous material looks like a "brick wall" with bricks sized on the order of several microns. Both pillars and bricks are composed of nanoparticles. The mechanical properties of the hierarchical biomaterial are measured by using the nanoindentation test. Hardness and modulus are measured for both the nacre layer and the prismatic layer, respectively. The nanoindentation size effects for the hierarchical structural materials are investigated experimentally. The results show that the prismatic nanostructured material has a higher stiffness and hardness than the nacre nanostructured material. In addition, the nanoindentation size effects for the hierarchical structural materials are described theoretically, by using the trans-scale mechanics theory considering both strain gradient effect and the surface/interface effect. The modeling results are consistent with experimental ones.
\end{abstract}

Keywords Biomaterial - Hierarchical structure - Mechanical property $\cdot$ Nanoindentation size effect . Trans-scale mechanics

\section{Yueguang Wei}

Ywei@LNM.imech.ac.cn

Jingru Song

Songjingru@LNM.imech.ac.cn

1 LNM, Institute of Mechanics, Chinese Academy of Sciences, Beijing 100190, China

\section{Introduction}

Mineralized biological materials, such as shells, bone, and teeth, have been attracting a great deal of attention during the last several decades [1-15], specifically, in more recent years [1-6], due to their unique microstructures and superior mechanical properties. The limnetic shell is a typical example of such biomaterial; it consists of nacreous and prismatic structures which display unique hierarchical features.

During the last several decades, much research on the mechanical behavior of mollusk shells have been focused on nacre structures. However, there is very little research on prismatic structures. A nacre structure has superior mechanical properties, because it is composed of $95 \%$ mineral and $5 \%$ organic matter (mainly protein and polysaccharides) [15]. Its stiffness is on the same order as mineral, but its strength is two to three times higher than that of mineral. Specifically, the fracture toughness of a nacre structure is 3000 times higher than that of a monolithic mineral [16-19]. Thus far, the mechanical properties of even man-made ceramic cannot reach such a high level $[20,21]$. Thus, much research on the strengthening-toughening mechanism of the nacre structure has been carried out. Currey first observed the staggered arrangement of nacre platelets and presented a "Brick and Mortar" model to describe the mechanical behavior of the nacre platelets [15]. Wang et al. [22,23] observed the nanoasperities on the platelets and developed a finite element (FE) model based on the friction mechanism. Song et al. [24,25] found the mineral bridges (which are mineral connections crossing a protein layer between adjacent mineral platelets) and proposed a "Brick, Bridge, and Mortar" model to interpret the strengthening mechanism of nacre structures. Katti et al. [26] developed a platelet interlock model. Barthelat et al. [27] observed a wavy structure and presented an FE model. 
In previous studies on the mechanical properties of nacre structures, tensile, compressive, and bending tests were used $[15,16,18,22,27]$. Jackson et al. [17] presented a criterion about the span-depth ratio of the beam specimens in the bending tests in order to obtain effective experimental results [17]; however, it was much more complex in the plastic case than the elastic case. Currey adopted the tensile test to study the mechanical behaviors of mother of pearl, and measured the load-deformation curve [15]. Barthelat et al. [27] conducted tensile tests; the specimen they used was so small (the parallel segment is $1.5 \mathrm{~mm}$ ) that the size effect was obvious in their results [27]. Zuo and Wei [28] investigated the mechanical behaviors of the limnetic shell. They observed and displayed the microstructure feature of nacre structures ("Brick and Mortar" structure) and measured the stressstrain relations by using both the three-point bending test and the tensile test. They modeled the mechanical properties of the nacre structure by using a modified shear-lag model.

Simultaneously, many studies on the mechanical properties of nacre structures used the nanoindentation test [29-34]. Wang et al. [29] studied a type of limnetic shell (Cristaria plicata) using the nanoindentation test and proposed several strengthening mechanisms based on their observations. They found that there were some distinctions between seashells and limnetic shell structures. Li et al. [30] investigated the mechanical properties of nacre structures for a type of red abalone by using the nanoindentation test; they measured the elastic modulus and hardness. Bruet et al. [31] used the nanoindentation test to measure the average elastic modulus and hardness for the nacre structure of a Trochus niloticus shell. Katti et al. [32] performed the nanoindentation test for measuring the mechanical properties of nacre platelets by using atomic force microscopy (AFM). In addition, Li et al. [30] and Sun et al. [33] used a microhardness tester with a Vickers tip to observe the crack propagation for nacre shell samples. Both microscale and nanoscale hardness were compared for five species of seashells which are of the different microstructures [34].

All research on the microstructure observation of the nacre platelets has shown that the nacre layer is a hierarchical structure. It is a "brick and mortar" structure at microor macroscopic scales, while the "brick" is composed of nanoparticles. The measured mechanical property, based on both the tensile test and the bend test, is on a macroscopic scale. However, the measured mechanical property based on the nanoindentation test is on a nanoscale. Strictly speaking, two types of properties measured based on the different scale test methods are not comparable, and there are huge difference among them. In order to describe the mechanical behavior for the hierarchical structure, Gao [35] developed a hierarchical model for biomaterials to try to connect the mechanical behaviors from nanoscale to macroscopic scale, implying a self-similarity assumption of microstructure. He studied the mechanical property transition from nanoscale to macroscopic scale for a hierarchical case of bone structure by using the tension-shear chain model [7]. Adopting the self-similarity assumption of microstructure, Zuo and Wei [36] investigated the trans-scale mechanical property of the hierarchical structure theoretically, by using the modified shear-lag model. In order to interpret the huge property difference between nanoscale and macroscopic scale, more recently, damage, flaw tolerances, and hierarchical features in the nacre structure were investigated [1-6].

Until now, most studies for mollusk shells using the nanoindentation test have focused only on the mechanical behaviors of the nacre platelets, and only in the direction perpendicular to the face of the nacre platelets.

In the present research, the hierarchical structures of both the nacre layer and prismatic layer for a kind of limnetic shell (Hyriopsis cumingii) will be observed and measured systematically by using scanning electron microscopy (SEM) and AFM. The nanoindentation size effects for the hierarchical structures of both nacre and prismatic layers will be measured by using the nanoindentation test. Nano/microscale hardness will be measured on the Nano Indenter G200 (Agilent Technologies). In addition, the nanoindentation size effects will be modeled by using the trans-scale mechanics theory considering both the strain gradient effect and surface/interface effect.

\section{Experimental observation and measurement}

\subsection{Microstructure feature}

The mollusk shell analyzed in this work is $H$. cumingii, which is a type of limnetic shell from the south of China. Microstructure observations are carried out by using SEM (JEOL JSM-7500F) and Veeco AFM. The general structure of the limnetic shell is shown in Fig. 1a, an SEM photo of a cross-section of the limnetic shell with a magnification 20 . The cross-section can be distinctly divided into two regions in the thickness direction according to the microstructure features: prism region and nacre region. It can be seen that the nacre region occupies about $80 \%$ of the total area. From Fig. 1a-1d and Fig. 1a, 1e, and 1f, both the prismatic structure and the nacre structure are hierarchical features from nanoscale to macroscopic scale. The nacre structure is the "brick and mortar" structure and the "brick" is composed of nanoparticles, while the prismatic structure is constructed of pillars of various sections, which is also composed of nanoparticles. The particle sizes making up the "brick" and "pillar" are about $30-40 \mathrm{~nm}$. The thickness of the "brick" is about $800-1100 \mathrm{~nm}$, while the height of the "pillar" is about 80-120 microns. From the vertical view, both the "brick" 


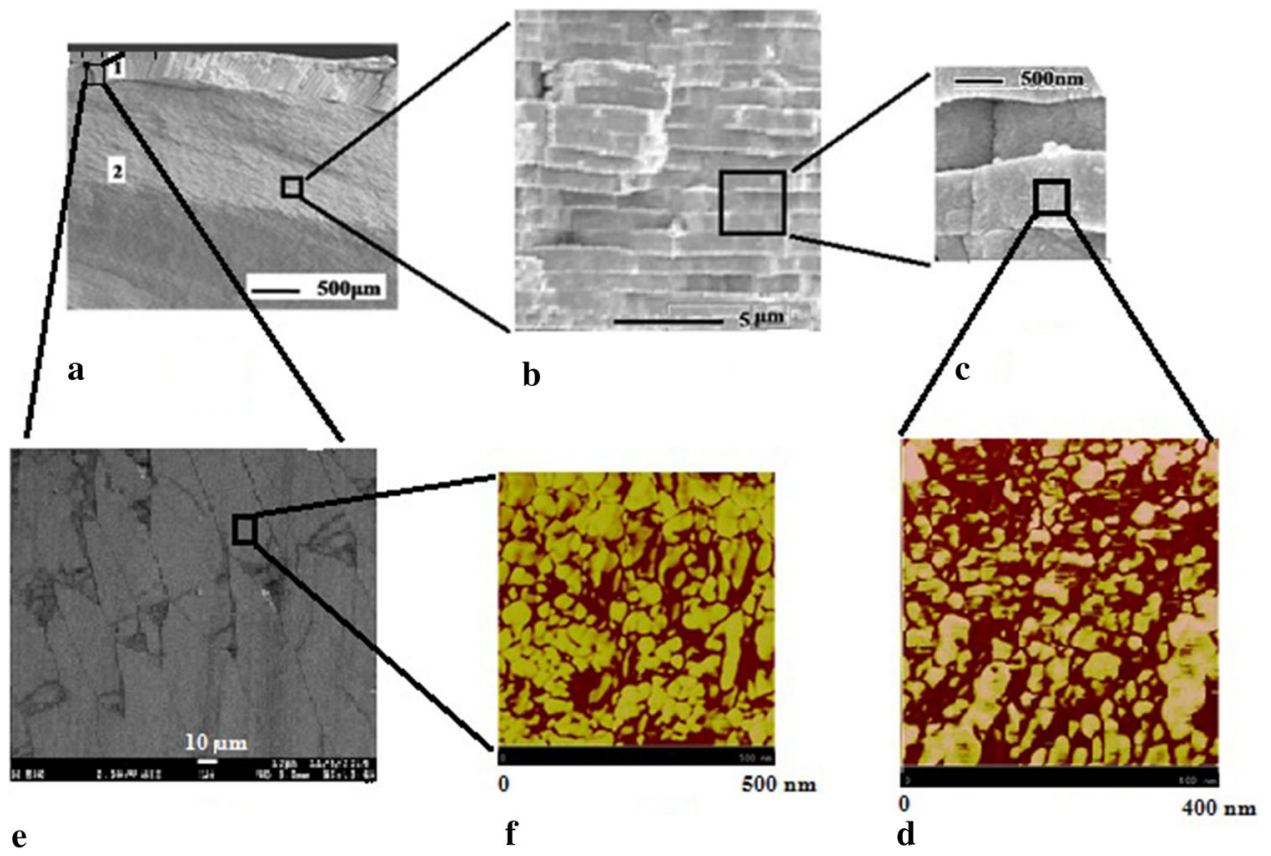

Fig. 1 Hierarchical structures of limnetic shell. a-d show the hierarchical structure of nacre layer. a, e and $\mathbf{f}$ show the hierarchical structure of prismatic layer

a

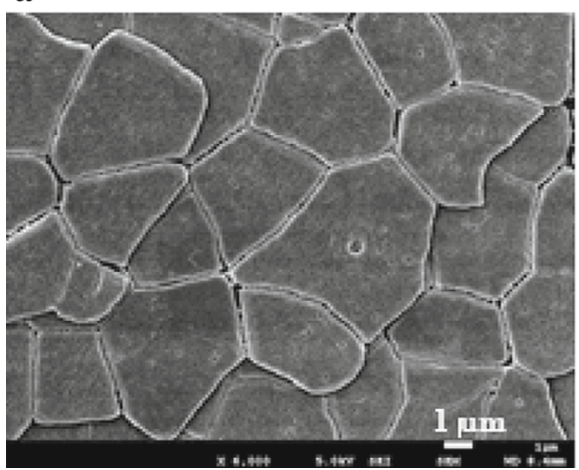

b

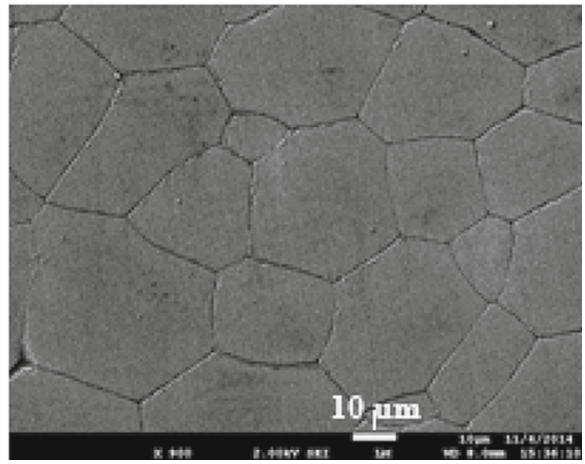

Fig. 2 SEM photos of nacre and prismatic structures from vertical view, a for nacre structure. $\mathbf{b}$ for prismatic structure

and "pillar" structures have hexagonal sections, as shown in Fig. 2. The diagonal of the hexagon for the nacre brick is about $5-10$ microns, while it is about $30-60$ microns for the prismatic pillar. Therefore, the average size of the cross-section of the pillar for the prismatic structure is much larger than the top surface size of the brick for the nacre structure.

\subsection{Hardness measurement}

In order to measure the hardness for both the nacre layer and the prismatic layer, samples are sectioned into parallelepipeds by using a water-cooled, low-speed diamond saw. For vertical view (perpendicular to the shell plane) SEM imaging, the samples were put into $10 \mathrm{wt} \%$ ethylene diaminetetra-acetic acid disodium salt (EDTA-2Na) solutions for
$10 \mathrm{~min}$, and for side view (parallel to the shell plane) SEM imaging, the samples were made in correspondence with a cutting surface, see Section A and Section B, respectively, in the sketched Fig. 3 for the brick and mortar structure of the nacre layer.

Samples were mechanically ground and polished using abrasives and rinsed thoroughly using an ultrasonic cleaner for nanoindentaion test convenience. They were put in ambient conditions prior to testing. Hardness measurements based on the nanoindentation test were performed using a Nano Indenter G200 (Agilent Technologies) with a diamond Berkovich tip (tip curvature radius is about $20 \mathrm{~nm}$ ) and a force resolution of about $1 \mathrm{nN}$ and displacement resolution about $0.0002 \mathrm{~nm}$.

The tests were carried out in displacement-control mode, and the maximum indentation depth was set to be $1 \mu \mathrm{m}$. After 


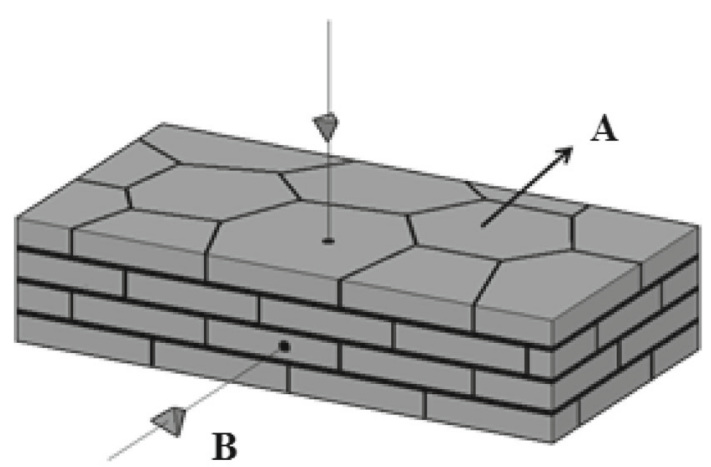

Fig. 3 Sketched figure for the indent section definitions of the nacre structure

tests, the indentation impressions were then imaged using Veeco AFM. For comparison, the hardness of prismatic layer material was also measured along the cross-section of the pillars (Section A) and the side surface of the pillars (Section B), respectively. Hardness $(H)$ and elastic modulus $(E)$ were determined using the Oliver-Pharr method [37] according to the following definitions:

$H=\frac{P}{A}, \quad \frac{1}{E_{\mathrm{r}}}=\frac{1-v^{2}}{E}+\frac{1-v_{\mathrm{i}}^{2}}{E_{\mathrm{i}}}$,

where $P$ is indent load, $A$ is the contact area, $E$ and $v$ are the elastic modulus and Poisson's ratio for the sample, respectively, $E_{\mathrm{i}}$ and $v_{\mathrm{i}}$ are the same quantities for the indenter. For the diamond indenter, $E_{\mathrm{i}}=1141 \mathrm{GPa}$ and $v_{\mathrm{i}}=0.07$. The reduced elastic modulus $\left(E_{\mathrm{r}}\right)$ is obtained using a relation involving contact stiffness and the contact area.

\section{Experimental results and discussions}

In the present research, the mechanical properties of both the nacre and prismatic layers were measured and compared. Hardness and elastic modulus were measured on two sections: Section A and Section B.

Figure 4 shows a representative indent impression on Section A of the nacre layer taken using Veeco AFM, and the indent impression is a three-square cone. Clearly, plastic deformation takes place for the "brick" under nanoindentation.

Figure 5 shows the representative hardness-depth curves of the nacreous structure with the maximum indentation depth at $1 \mu \mathrm{m}$. Results shown in Fig. 5a and 5b correspond to the cases of the nanoindentation on Section A and Section $\mathrm{B}$, respectively. Each curve in the figures corresponds to each indent point on the section. Each figure includes ten curves which correspond to ten indented points on the section, which are selected randomly. It can be seen that the hardness values

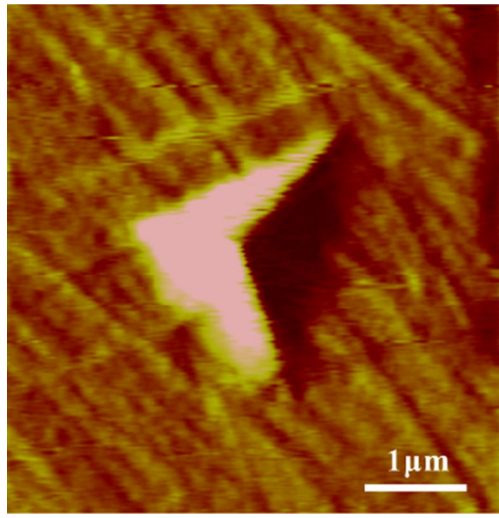

Fig. 4 AFM photo of a representative indent impression on Section A of the nacre layer

vary continuously with the increase of the indent depth. At the test beginning and at very shallow indent depth, the hardnessdepth curves have a severe variation, which could be caused by many factors, including the unsteady contact between the indenter and the sample surface at the test beginning, surface profiles of the samples and indenter, etc. However, the hardness-depth curves tend to stable quickly.

As shown in Fig. 5, usually the experimental hardnessdepth curve is not monotonically decreasing with depth increase; this is because the indenter tip is not very sharp and has a curvature radius. The curvature radius for the Nano Indenter G200 is about $20 \mathrm{~nm}$. However, the hardness-depth law (indentation size effect) is less influenced by the curvature radius when the indent depth is larger than about $50 \mathrm{~nm}$.

From Fig. 5a and 5b, a common feature is obvious: when the indent depth is larger than about $50 \mathrm{~nm}$, hardness values increase with decreasing the indent depth, which is an obvious nanoindentation size effect. Compared to the hardness-depth curves given in Fig. 5a with Fig. 5b, one can find that the measured hardness value for Section $\mathrm{A}$ is larger than that for Section B, possibly because there is a stronger constraint from interface to resist the force from the vertical direction on Section A. We use our previous research conclusion to interpret the findings on the hardness-depth relation for nanocrystalline metals and for a metal film/ceramic substrate system; the interface (grain boundary) constraint is a major effect on the hardness-depth relations [38].

In order to compare the mechanical properties on both Section A and Section B for the nacre structure, we further take the average hardness based on the experimental results shown in Fig. 5a and 5b and show the results in Fig. 6a and 6b, respectively. Obviously, the average hardness of Section A is larger than of Section B. However, when we take an average for the elastic modulus, the conclusion is the opposite; that is to say, the average modulus measured for Section A is smaller than that for Section B. The results for both sections are also shown in Fig. 6a and 6b, respectively. 


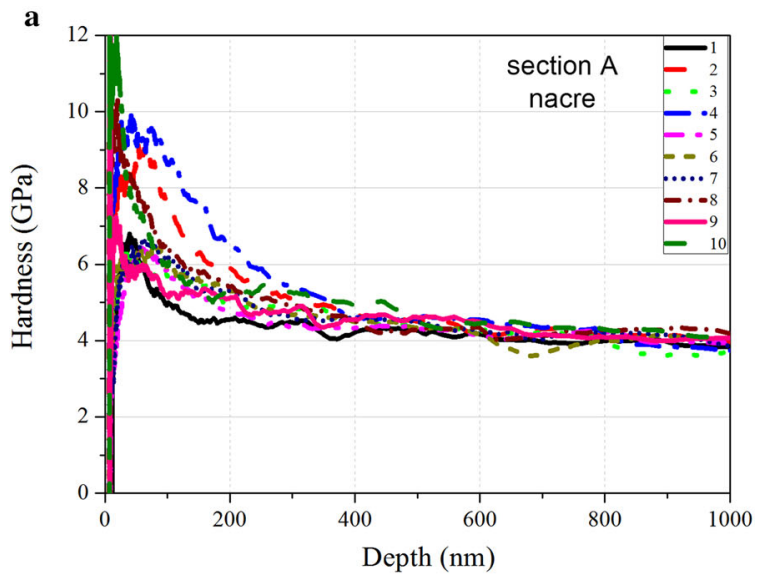

b

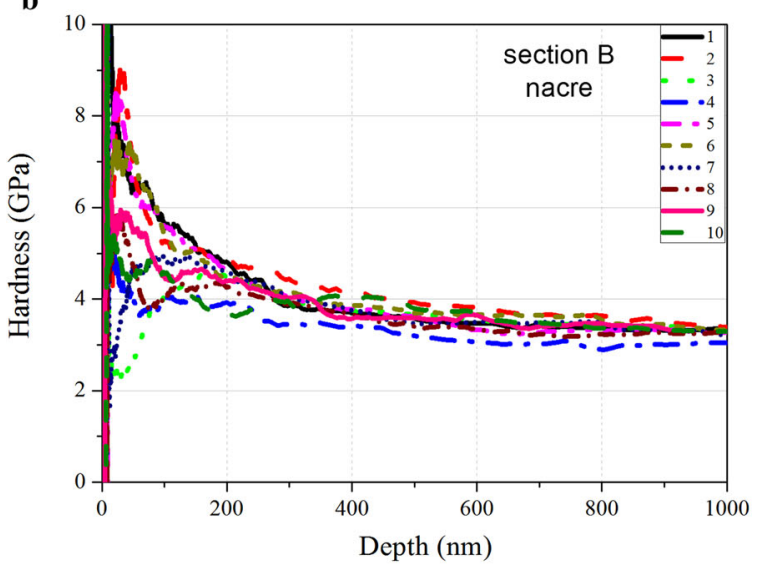

Fig. 5 Hardness-depth curves of the nacre structure corresponding to ten indent points on sections, $\mathbf{a}$ for Section A and $\mathbf{b}$ for Section B

It is interesting to compare the mechanical properties for both nacre structure and prismatic structure. We have measured hardness values for the prismatic structure along its Section A and Section B, respectively, where Section A corresponds to its hexagonal sections (see Fig. 2b), while Section $\mathrm{B}$ corresponds to the side section (see Fig. 1e). The average hardness results for the prismatic structure on Section A and Section B are shown in Fig. 7a and 7b, respectively. The conclusions to compare the results of both Section A and Section $\mathrm{B}$ for the prismatic structure are same as for the nacre structure. However, through comparing the mechanical properties for both nacre structure (Fig. 6) and the prismatic structure (Fig. 7), one can find that both hardness and modulus measured for the prismatic structure are obviously larger than the correspondences of the nacre structure.

\section{Modeling and simulation of nanoindentation hardness for the limnetic shell}

Most biomaterials are hierarchical structures. The limnetic shell (H. cumingii) is a representative hierarchical structure. From Fig. 1, the limnetic shell consists of a nacre layer and
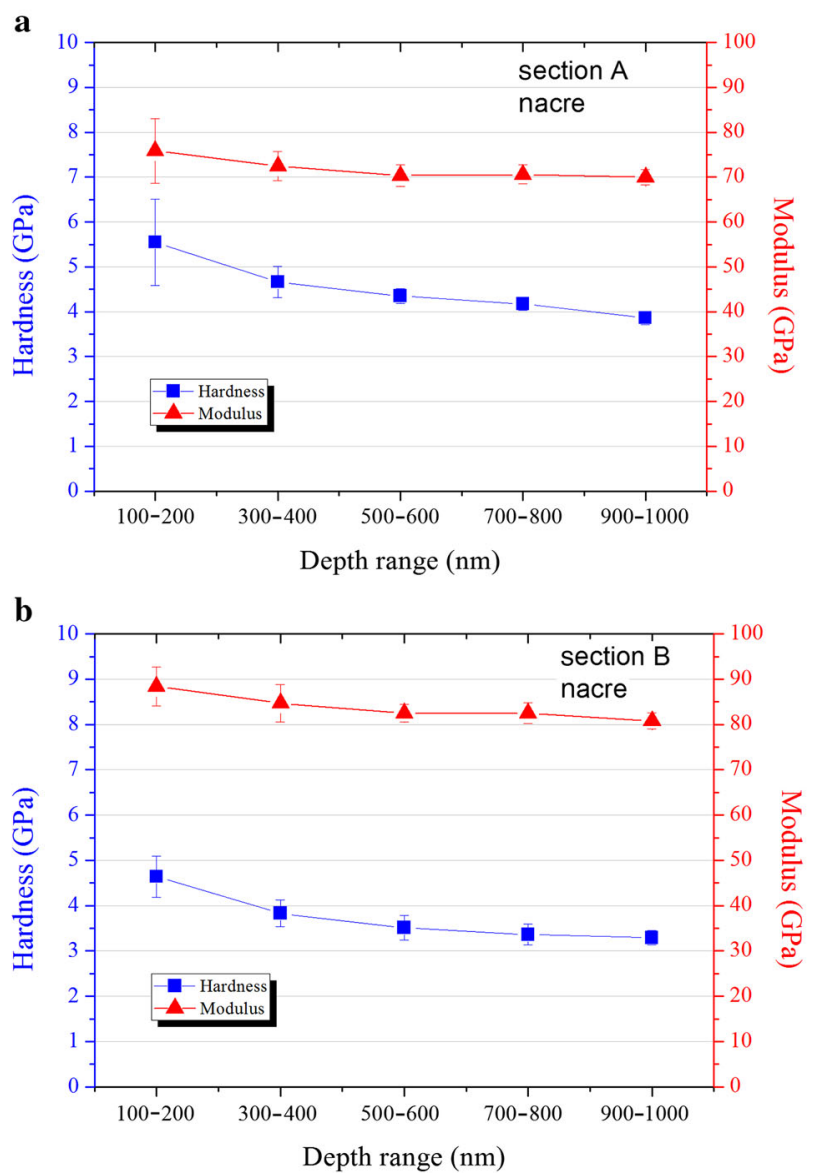

Fig. 6 For the nacre structure, measured average hardness/modulus values for different depth ranges based on ten indent points, a for Section $\mathrm{A}$ and $\mathbf{b}$ for Section $\mathrm{B}$

a prismatic layer, and each material region is a hierarchical structure. In the above experimental measurement and analyses, we systematically display the mechanical properties for the hierarchical structures through measuring hardness variations at nano- or microscales using the nanoindentation test. The experimental results show a strong nanoindentation size effect. In the present section, we intend to describe the nanoindentation size effect of the hierarchical structure by using the trans-scale mechanics theory considering both strain gradient effect and the surface/interface effect. The used strain gradient plasticity theory is Wei-Hutchinson's version $[39,40]$, which can be used in a wide variety of materials, and is not limited to the cases of the typical metal materials like other versions which were built up specifically based on the dislocation concepts.

\subsection{A brief introduction to the trans-scale mechanics theory}

For trans-scale mechanics theory considering both strain gradient effect and surface/interface effects, a variation equation 


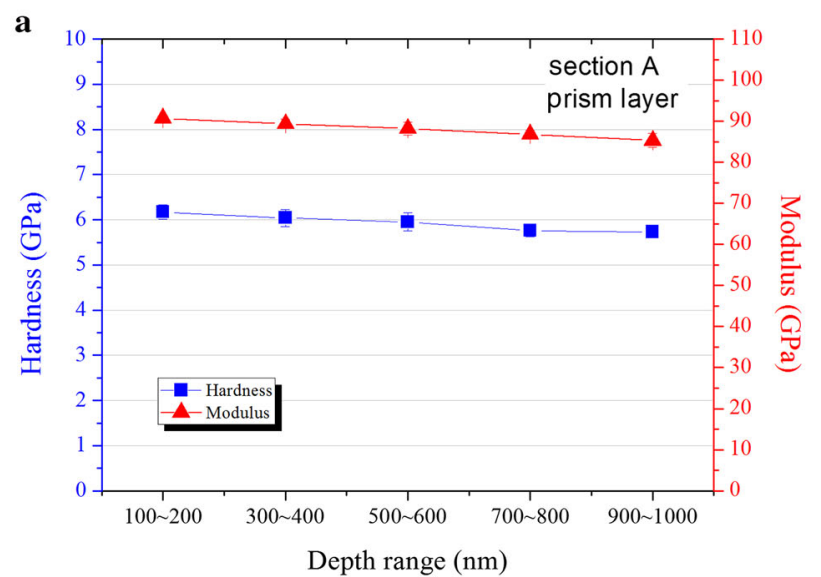

b

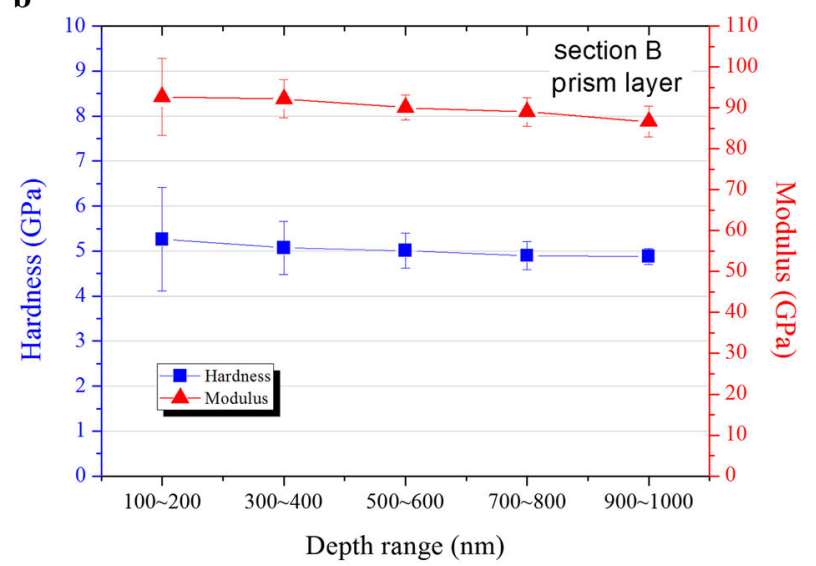

Fig. 7 For the prismatic structure, measured average hardness/modulus values for different depth range based on ten indent points, $\mathbf{a}$ for Section A and $\mathbf{b}$ for Section B

of total potential can be expressed as follows $[41,42]$

$$
\begin{gathered}
\int_{V}(\boldsymbol{\sigma} \delta \boldsymbol{\varepsilon}+\boldsymbol{\tau} \delta \boldsymbol{\eta}) \mathrm{d} V+\delta \int_{S^{\prime}} \gamma \mathrm{d} S^{\prime}-\int_{V} \overline{\boldsymbol{f}} \delta \boldsymbol{u} \mathrm{d} V \\
-\int_{S}\left[\overline{\boldsymbol{t}} \delta \boldsymbol{u}+\overline{\boldsymbol{S}}_{0}^{s} \delta \boldsymbol{\varepsilon}^{s}+\overline{\boldsymbol{r}} \cdot(\delta \boldsymbol{u} \boldsymbol{n} \cdot \nabla)\right] \mathrm{d} S=0,
\end{gathered}
$$

where $\tau$ and $\eta$ are the tensors of higher-order stress and strain gradient, $\boldsymbol{\tau} \delta \boldsymbol{\eta}$ is the variation of strain energy density contributed by the strain gradient effect, $\overline{\boldsymbol{r}} \cdot(\delta \boldsymbol{u} \boldsymbol{n} \cdot \nabla)$ is surface specific work done by higher-order stress moment, $\gamma$ is the surface/interface energy density, $\delta \int_{S^{\prime}} \gamma \mathrm{d} S^{\prime}$ is the variation of surface/interface energy calculated along transient surface area, $\overline{\boldsymbol{S}}_{0}^{\mathrm{s}} \delta \boldsymbol{\varepsilon}^{\mathrm{s}}$ is surface/interface residual work.

In Eq. (2), the small strain case is considered. Specifically, for surface/interface energy, its total contribution comes from two parts: one part is from the variation of the surface/interface energy density itself, another is from variation of the surface/interface area. The surface/interface energy density consists of two parts: one part is residual energy density and another part comes from surface/interface strain. Therefore, for the small strain case, the calculation of total surface/interface energy should be based on the present domain $\left(S^{\prime}\right)$ instead of the reference domain $(S)$. When representative cell size of a solid is at the micrometer scale, the surface/interface effect is small and can be neglected relative to the strain gradient effect; however, when it is at the nanometer scale, the surface/interface effect is important and can't be neglected to compare with strain gradient effect. For the present case, when describing the nanoindentation hardness of hierarchical structure, both strain gradient effect and the surface/interface effect are important.

In Eq. (2), the adopted constitutive equations of the strain gradient theory are Wei-Hutchinson's version expressed in the component form as follows $[39,40]$ :

$$
\begin{aligned}
\sigma_{i j}= & \frac{E}{1+v+\frac{3}{2} E / h^{\mathrm{p}}} \varepsilon_{i j} \\
& +\frac{1}{3}\left(\frac{E}{1-2 v}-\frac{E}{1+v+\frac{3}{2} E / h^{\mathrm{p}}}\right) \varepsilon_{k k} \delta_{i j} \\
\tau_{i j k}= & 2 E \ell^{2}\left(\sum_{I=1}^{3} \frac{1}{1+2 E / h^{\mathrm{p}}} T_{i j k l m n}^{(I)}+T_{i j k l m n}^{(4)}\right) \eta_{l m n}, \\
h^{\mathrm{p}}= & E\left[\left(\Sigma / \sigma_{\mathrm{Y}}\right)^{1 / N-1}-1\right]^{-1}
\end{aligned}
$$

where $\boldsymbol{T}$ is the projection tensor of the higher-order stresses, $h^{\mathrm{p}}$ is the plastic hardening modulus, $\Sigma$ is the effective stress, $\ell$ is material length scale which describes that strain gradient effect becomes important when the scale of nonuniform straining becomes of order $\ell, \sigma_{\mathrm{Y}}$ is material yield strength, $v$ is Poisson's ratio, $N$ is strain hardening exponent.

In Eq. (2), considering the small strain case and considering the current configuration in calculating the surface/interface energy, the surface/interface constitutive equations can be expressed as follows [42]:

$\boldsymbol{S}^{\mathrm{S}}=J^{\mathrm{S}} \boldsymbol{f}^{\mathrm{S}}+\gamma \boldsymbol{I}^{\mathrm{S}}, \boldsymbol{f}^{\mathrm{S}}=\frac{\partial \gamma}{\partial \boldsymbol{\varepsilon}^{\mathrm{S}}}, J^{\mathrm{S}}=1+\boldsymbol{\varepsilon}^{\mathrm{S}}: \boldsymbol{I}^{\mathrm{S}}$,

where $\left(\boldsymbol{S}^{\mathrm{S}}, \boldsymbol{\varepsilon}^{\mathrm{S}}\right)$ is the surface/interface stress and strain, $\boldsymbol{I}^{\mathrm{S}}$ is the surface/interface unit tensor, $J^{\mathrm{S}}$ is a Jacobi coefficient of the surface/interface area, $\gamma=\left(\gamma_{\mathrm{s}}, \Gamma\right)$ is the surface energy density $\left(\gamma_{\mathrm{s}}\right)$ or interface energy density $(\Gamma)$. Considering a simple case, $\gamma\left(\varepsilon^{\mathrm{S}}\right)=$ constant, Eq. (4) can be simplified as

$S^{\mathrm{S}}=\gamma \boldsymbol{I}^{\mathrm{S}}$.

\subsection{Characterization of nanoindentation size effect}

Based on the above trans-scale mechanics theory considering both strain gradient effect and the surface/interface effect, 
and considering the case in Eq. (5), the relationship between the nanoindentation hardness with indent depth for a circular conic indenter case and for the hierarchical materials can be expressed in a non-dimensional form as follows:

$\frac{H}{3 \sigma_{\mathrm{Y}}}=f\left(\frac{h}{\ell}, \frac{E}{\sigma_{\mathrm{Y}}}, v, N, \beta, \frac{\gamma_{s}}{\sigma_{\mathrm{Y}} h}, \frac{\Gamma}{\sigma_{\mathrm{Y}} d_{0}}\right)$,

where $h$ is indent depth, $d_{0}$ is the particle size of the composed "brick" for the nacre structure, $\beta$ is the surface slope angle to the horizontal plane for the circular conic indenter.

The hardness-depth relation Eq. (6) can be obtained through FE modeling based on the trans-scale mechanics theory from Eqs. (2-4) or (5), and the feature of the experimental hardness-depth curves shown in Fig. 5 can be also simulated by using the trans-scale mechanics theory through considering a curvature radius existing at indenter tip. In the present research, we use a simple method to obtain the solution for Eq. (6). Through a quantity level analysis, the dimensionless quantities both $\gamma_{s} / \sigma_{\mathrm{Y}} h$ and $\Gamma / \sigma_{\mathrm{Y}} d_{0}$ are smaller than unity, thus the hardness-depth solution Eq. (6) can be approximately expressed as follows through small parameter expansion:

$$
\begin{aligned}
\frac{H}{3 \sigma_{\mathrm{Y}}} \approx & g_{0}\left(\frac{h}{\ell}, \frac{E}{\sigma_{\mathrm{Y}}}, v, N, \beta\right)+\frac{2}{3 \cos \beta} \frac{\gamma_{s}}{\sigma_{\mathrm{Y}} h} \\
& +\frac{2 \sqrt{3} \Gamma}{\sigma_{\mathrm{Y}} d_{0} \tan \beta}\left(1-\frac{d_{0} \tan \beta}{2 \sqrt{3} h}\right),
\end{aligned}
$$

where $g_{0}$ is the solution based on only the strain gradient effect, and other terms are the contributions due to the surface/interface effects. Solution (7) is based on an assumption that the particles are all of the same cubic shape and size $d_{0}$.

The hardness-indent depth relations considering only strain gradient effect were investigated by Wei et al. [43], and the solution form is as follow:

$$
\frac{H_{\mathrm{SGP}}}{3 \sigma_{\mathrm{Y}}}=g_{0}\left(\frac{h}{\ell}, \frac{E}{\sigma_{\mathrm{Y}}}, v, N, \beta\right),
$$

which is re-plotted in Fig. 8.

For the nanoindentation of the hierarchical materials, combining the hardness-indent depth solution based on the strain gradient theory (Eq. 8) shown in Fig. 8, with the surface/interface effect, one can obtain the full solution details for Eq. (7). Using the modeling results to simulate the experimental results (for example, Fig. 5b) of nanoindentation hardness for the nacre structure, the simulation results are shown in Fig. 9. In Fig. 9, two experimental hardness curves correspond to the highest and lowest curves given in Fig. 5b, respectively. From Fig. 9, the parameter values taken for the theoretical solutions are reasonable, and the trans-scale theory can give an effective modeling for

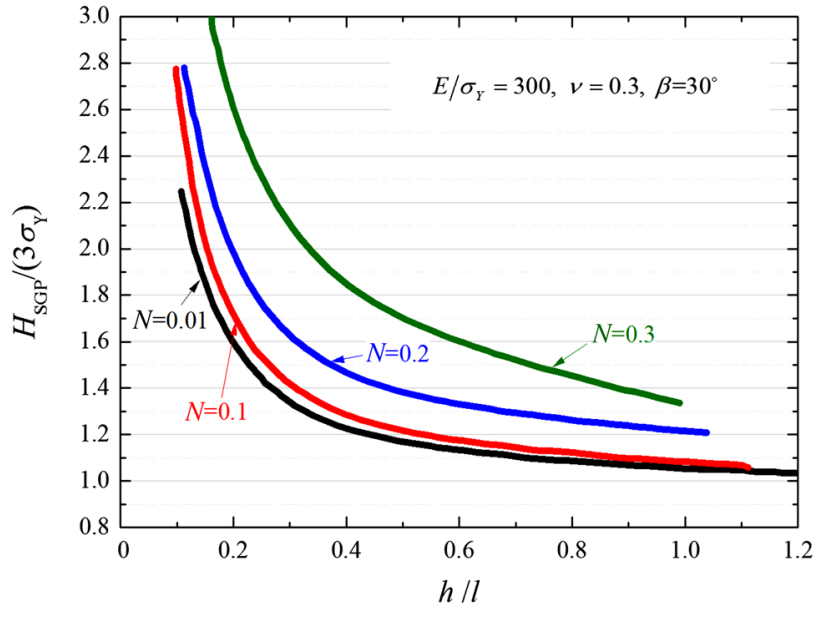

Fig. 8 Normalized hardness depth relations modeled based on the strain gradient plasticity theory (see Ref. [43])

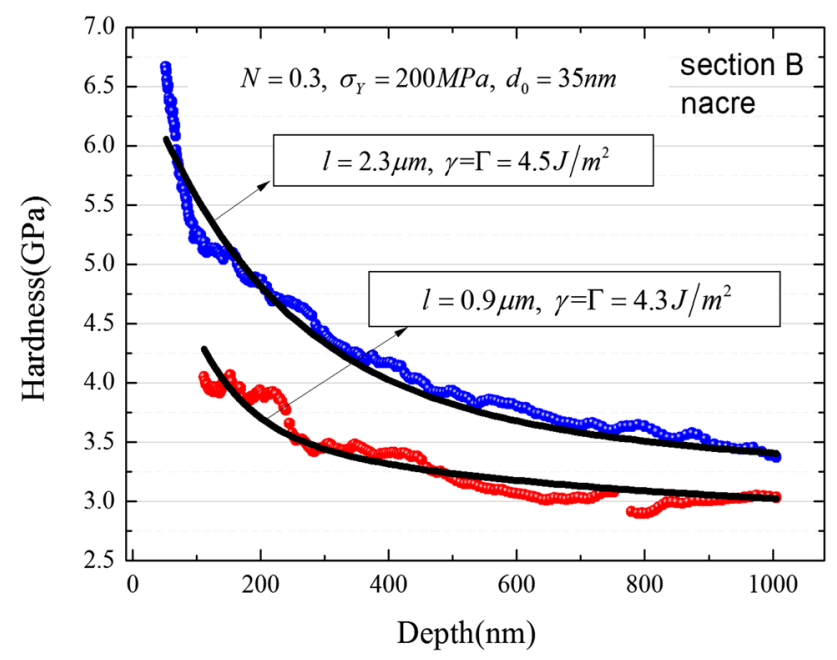

Fig. 9 Simulations to experimental results. Two experimental curves correspond to highest and lowest curves in Fig. 5b, respectively. Theoretical results are based on the trans-scale mechanics theory

the nanoindentation size effect of the hierarchical biomaterials.

\section{Conclusions}

Microstructure observation and mechanical property measurement for a type of biomaterial have been performed. The measured biomaterial is $H$. cumingii, a typical limnetic shell, which is consisted of two different structural layers, the prismatic "pillar" structure and the nacreous "brick and mortar" structure. Microstructure materials in each layer display the hierarchical feature clearly. The prismatic layer is composed of a variation-section pillar with size several tens of microns, and each pillar is composed of nanoparticles. The nacre layer 
is composed of a "brick wall" structure with bricks sized several microns, and each brick is composed of nanoparticles.

The mechanical properties of the hierarchical biomaterial have been measured by using the nanoindentation test. Nanoindentation hardness and modulus have been measured for the nacre layer ("brick and mortar" structure) and the prismatic layer ("pillar" structure), respectively. The results have shown that the prismatic nanostructured material has a bigger stiffness and hardness than the nacre nanostructured material. Hardness-indent depth curves for both nanostructured material regions have shown strong nanoindentation size effects.

The nanoindentation size effects of hardness for the hierarchical biomaterial ("brick and mortar" structure of the nacre layer) have been modeled by using the trans-scale mechanics theory considering both strain gradient effect and the surface/interface effect. From modeling results, the transscale mechanics theory is sufficiently effective to be used to describe the nanoindentation size effect of hardness for hierarchical materials, keeping a reasonable selection of the material parameters.

\section{References}

1. Xie, Z.Q., Yao, H.M.: Crack deflection and flaw tolerance in "brickand-mortar" structured composites. Int. J. Appl. Mech. 6, 1450017 (2014)

2. Shao, Y., Zhao, H.P., Feng, X.Q.: On flaw tolerance of nacre: a theoretical study. J. R. Soc. Interface 11, 20131016 (2014)

3. Xu, J., Zhao, X.L., Munroe, P., et al.: Synergistic toughening of hard, nacre-mimetic MoSi2 coatings by self-assembled hierarchical structure. Sci. Rep. 4, 4239 (2014)

4. Brandt, K., Wolff, M.F.H., Salikov, V., et al.: A novel method for a multi-level hierarchical composite with brick-and-mortar structure. Sci. Rep. 3, 2322 (2013)

5. Ji, B.H., Gao, H.J.: Mechanical principles of biological nanocomposites. Annu. Rev. Mater. Res. 40, 77-100 (2010)

6. Bechtle, S., Ang, S.F., Schneider, G.A.: On the mechanical properties of hierarchically structured biological materials. Biomaterial 31, 6378-6385 (2010)

7. Ji, B.H., Gao, H.J.: Mechanical properties of nanostructure of biological materials. J. Mech. Phys. Solids 52, 1963-1990 (2004)

8. Weiner, S., Veis, A., Beniash, E., et al.: Peritubular dentin formation: crystal organization and the macromolecular constituents in human teeth. J. Struct. Biol. 126, 27-41 (1999)

9. Tesch, W., Eidelman, N., Roschger, P., et al.: Graded microstructure and mechanical properties of human crown dentin. Calcif. Tissue Int. 69, 147-157 (2001)

10. Landis, W.J.: The strength of a calcified tissue depends in part on the molecular structure and organization of its constituent mineral crystals in their organic matrix. Bone 16, 533-544 (1995)

11. Rho, J.Y., Kuhn-Spearing, L., Zioupos, P.: Mechanical properties and the hierarchical structure of bone. Med. Eng. Phys. 20, 92-102 (1998)

12. Weiner, S., Wagner, H.D.: The material bone: structure-mechanical function relations. Annu. Rev. Mater. Sci. 28, 271-298 (1998)

13. Taylor, J.D., Layman, M.: The mechanical properties of bivalve (Mollusca) shell structures. Palaeontology 15, 73-87 (1972)
14. Currey, J.D., Taylor, J.D.: The mechanical behaviour of some molluscan hard tissues. J. Zool. 173, 395-406 (1974)

15. Currey, J.D.: Mechanical properties of mother of pearl in tension. Proc. R. Soc. Lond. B 196, 443-463 (1977)

16. Kamat, S., Su, X., Ballarini, R., et al.: Structural basis for the fracture toughness of the shell of the conch Strombus gigas. Nature 405, 1036-1040 (2000)

17. Jackson, A.P., Vincent, J.F.V., Turner, R.M.: The mechanical design of nacre. Proc. R. Soc. Lond. B 234, 415-440 (1988)

18. Menig, R., Meyers, M.H., Meyers, M.A., et al.: Quasi-static and dynamic mechanical of haliotis rufescens (abalone) shells. Acta Mater. 48, 2383-2398 (2000)

19. Menig, R., Meyers, M.H., Meyers, M.A., et al.: Quasi-static and dynamic mechanical response of Strombus gigas (conch) shells. Mater. Sci. Eng. A 297, 203-211 (2001)

20. Almqvist, N., Thomson, N.H., Smith, B.L., et al.: Methods for fabricating and characterizing a new generation of biomimetic materials. Mater. Sci. Eng. C 7, 37-43 (1999)

21. Jackson, A.P., Vincent, J.F.V.: Comparason of nacre with other ceramic composites. J. Mater. Sci. 25, 3173-3178 (1990)

22. Wang, R.Z., Suo, Z., Evans, A.G., et al.: Deformation mechanisms in nacre. J. Mater. Res. 16, 2485-2493 (2001)

23. Evans, A.G., Suo, Z., Wang, R.Z., et al.: Model for the robust mechanical behavior of nacre. J. Mater. Res. 16, 2475-2484 (2001)

24. Song, F., Soh, A.K., Bai, Y.L.: Structural and mechanical properties of the organic matrix layers of nacre. Biomaterials 24, 3621-3631 (2003)

25. Song, F., Bai, Y.L.: Effects of Nanstructures on the fracture strength of the interfaces in nacre. J. Mater. Res. 18, 1741-1744 (2003)

26. Katti, K.S., Katti, D.R., Pradhan, S.M., et al.: Platelet interlocks are the key to toughness and strength in nacre. J. Mater. Res. 20, 1097-1100 (2005)

27. Barthelata, F., Tang, H., Zavattieri, P.D., et al.: On the mechanics of mother-of-pearl: a key feature in the material hierarchical structure. J. Mech. Phys. Solids 55, 306-337 (2006)

28. Zuo, S.C., Wei, Y.G.: Microstructure observation and mechanical behavior modeling for limnetic nacre. Acta Mech. Sin. 24, 83-89 (2008)

29. Wang, R.Z., Wen, H.B., Cui, F.Z., et al.: Observation of damage morphologies in nacre during deformation and fracture. J. Mater. Sci. 30, 2299-2304 (1995)

30. Li, X.D., Chang, W.C., Chao, Y.J., et al.: Nanoscale structural and mechanical characterization of a natural nanocomposite material: the shell of red abalone. Nano Lett. 4, 613-7 (2004)

31. Bruet, B.J.F., Qi, H.J., Boyce, M.C., et al.: Nanoscale morphology and indentation of individual nacre tablets from the gastropod mollusc Trochus niloticus. J. Mater. Res. 20, 2400-19 (2005)

32. Katti, K.S., Mohanty, B., Katti, D.R.: Nanomechanical properties of nacre. J. Mater. Res. 21, 1237-1242 (2006)

33. Sun, J.Y., Tong, J.: Fracture toughness properties of three different biomaterials measured by nanoindentation. J. Bionic Eng. 4, 11-17 (2007)

34. Fleischli, F.D., Dietiker, M., Borgia, C., et al.: The influence of internal length scales on mechanical properties in natural nanocomposites: a comparative study on inner layers of seashells. Acta Biomater. 4, 1694-1706 (2008)

35. Gao, H.J.: Application of fracture mechanics concepts to hierarchical biomechanics of bone and bone-like materials. Int. J. Fract. 138, 101-137 (2006)

36. Zuo, S.C., Wei, Y.G.: Effective elastic modulus of bone-like hierarchical materials. Acta Mech. Solida Sin. 20, 198-205 (2007)

37. Oliver, W.C., Pharr, G.M.: An improved technique for determining hardness and elastic-modulus using load and displacement sensing indentation experiments. J. Mater. Res. 7, 1564-83 (1992)

38. Wei, Y.G., Xu, G.: A multiscale model for the ductile fracture of crystalline materials. Int. J. Plast. 21, 2123-2149 (2005) 
39. Wei, Y.G., Hutchinson, J.W.: Steady-state crack growth and work of fracture for solids characterized by strain gradient plasticity. J. Mech. Phys. Solids 45, 1253-1273 (1997)

40. Wei, Y.G.: A new finite element method for strain gradient theories and applications to fracture analyses. Eur. J. Mech. A/Solids 25, 897-913 (2006)

41. Wu, B., Liang, L.H., Ma, H.S., et al.: A trans-scale model for size effects and intergranular fracture in nanocrystalline and ultra-fine polycrystalline metals. Comput. Mater. Sci. 57, 2-7 (2012)
42. Song, J.R., Liu, J.Y., Ma, H.S., et al.: Determinations of both length scales and surface elastic parameters for fcc metals. C. R. Mecanique 342, 315-325 (2014)

43. Wei, Y.G., Wang, X.Z., Wu, X.L., et al.: Theoretical and experimental researches of size effect in micro-indentation test. Sci. China Ser. A 44, 74-82 (2001) 